\title{
Implementation of a Protocol to Manage Patients at Risk for Hospitalization Due to an Ambulatory Care Sensitive Condition
}

\author{
Catherine M. Kuecker, PharmD, BCACP; Anita Kashyap, PharmD, BCACP; and Ellina Seckel, PharmD, BCACP, DPLA
}

\begin{abstract}
Background: Ambulatory care sensitive conditions (ACSCs), such as type 2 diabetes mellitus, chronic obstructive pulmonary disease, hypertension, congestive heart failure, urinary tract infections, asthma, dehydration, bacterial pneumonia, angina without an in-hospital procedure, and perforated appendix put patients at risk for hospitalization. Currently at the William S. Middleton Memorial Veterans Hospital in Madison, Wisconsin, no standardized process or protocol exists that can identify and optimize primary care for patients with ACSCs who have been hospitalized but are predicted to be at low risk for rehospitalization.
\end{abstract}

Methods: This project aimed to evaluate the implementation of offering further referrals and care for these patients. A pharmacy resident conducted a baseline chart review using a standardized template in the US Department of Veterans Affairs
(VA) Computerized Patient Record System to identify additional referrals or interventions a patient may benefit from based on any identified ACSC. Potential referral options included a clinical pharmacy specialist or nurse care manager disease management, whole health/wellness, educational classes, home monitoring equipment, specialty clinics, nutrition, cardiac or pulmonary rehabilitation, social work, and mental health.

Results: Comparing the 3 months prior to and the 3 months after offering referrals, there was a cumulative quantitative decrease in the number of emergency department visits (5 to 1) and hospitalizations (11 to 5).

Conclusions: Identifying patients at risk for hospitalization from an ACSC via a review and referral process by using the VA patient aligned care team structure was feasible and led to increased patient access to primary care and additional services.

\section{Catherine Kuecker and} Anita Kashyap are Clinical Pharmacy Specialists; Ellina Seckel is Associate Chief of Pharmacy, Ambulatory and Specialty Care; all at the William S. Middleton Memorial Veterans Hospital in Madison, Wisconsin. Correspondence: Catherine Kuecker (catherine.kuecker@va.gov)

Fed Pract. 2020;37(8):380-383. doi:10.12788/fp.0030
$\mathrm{H}$ ospitalizations related to ambulatory care sensitive conditions (ACSCs) are potentially avoidable if timely and effective care is provided to the patient. The Agency of Healthcare Research and Quality has identified type 2 diabetes mellitus (T2DM), chronic obstructive pulmonary disease (COPD), hypertension, congestive heart failure (CHF), urinary tract infections (UTIs), asthma, dehydration, bacterial pneumonia, angina without an inhospital procedure, and perforated appendix as ACSCs. ${ }^{1,2}$ Identifying patients with ACSCs who are at risk for hospitalization is a potential measure to enhance primary care delivery and reduce preventable hospitalizations.

The US Department of Veterans Affairs (VA) Clinical Pharmacy Practice Office implemented a guidance statement describing the role and impact of a clinical pharmacy specialist (CPS) in managing ACSCs. ${ }^{1}$ Within the Veterans Health Administration, the CPS may function under a scope of practice within their area of expertise with the ability to prescribe medications, place consults, and order laboratory tests and ad- ditional referrals as appropriate. As hospitalizations related to ACSCs are potentially preventable with effective primary care, the CPS can play an essential primary care role to implement interventions targeted at reducing these hospitalizations.

At the William S. Middleton Memorial Veterans Hospital, in Madison, Wisconsin, multiple transitions of care and postdischarge services have been established to capture those patients who are at a high risk of rehospitalization. Studies have been completed regarding implementation of intensive case management programs for high-risk patients. ${ }^{3}$ Currently though, no standardized process or protocol exists that can identify and optimize primary care for patients with ACSCs who have been hospitalized but are predicted to be at low risk for rehospitalization. Although these patients may not require intensive case management like that of those at high risk, improvements can be made to optimize clinical resources, education, and patient self-monitoring to mitigate risk for hospitalization or rehospitalization. Therefore, this project aimed to evaluate the 
implementation of offering further referrals and care for patients who have been hospitalized but are considered low risk for hospitalization from ACSCs.

\section{METHODS}

This quality improvement project to offer further referrals and care to patients considered low risk for hospitalization was implemented to enhance ambulatory-care provided services. All patients identified as being a low risk for hospitalization via a VA dashboard from July through September 2018 were included. Patients were identified based on age, chronic diseases, gender, and other patient-specific factors predetermined by the VA dashboard algorithm. Patients receiving hospice or palliative care and those no longer receiving primary care through the facility were excluded.

A pharmacy resident conducted a baseline chart review using a standardized template in the computerized patient record system (CPRS) to identify additional referrals or interventions a patient may benefit from based on any identified ACSC. Potential referral options included a CPS or nurse care manager disease management, whole health/wellness, educational classes, home monitoring equipment, specialty clinics, nutrition, cardiac or pulmonary rehabilitation, social work, and mental health. A pharmacy resident or the patient aligned care team (PACT) CPS reviewed the identified referrals with PACT members at interdisciplinary team meetings and determined which referrals to offer the patient. The pharmacy resident or designated PACT member reached out to the patient via telephone or during a clinic visit to offer and enter the referrals. If the patient agreed to any referrals, a chart review was conducted 3 months later to determine the percentage of initially agreed-upon referrals that the patient completed. Additionally, the number of emergency department (ED) visits and hospitalizations related to an ACSC at 3 months was collected.

Feasibility was assessed to evaluate potential service implementation and was measured by the time in minutes to complete the baseline chart review, time in minutes to offer referrals to the patient, and
TABLE Baseline Characteristics

\begin{tabular}{lc} 
Characteristics & Results (N = 69) \\
\hline Age, mean (SD), y & $67(11)$ \\
\hline $\begin{array}{l}\text { Sex, No. (\%) } \\
\text { Male }\end{array}$ & $66(96)$ \\
Female & $3(4)$ \\
\hline $\begin{array}{l}\text { Race, No. (\%) } \\
\text { White }\end{array}$ & $67(98)$ \\
African American & $1(1)$ \\
Asian & $1(1)$ \\
\hline $\begin{array}{l}\text { Cumulative hospitalizations, No. } \\
3 \text { mo prior to review }\end{array}$ & 11 \\
12 mo prior to review & 81 \\
\hline Cumulative emergency department visits, No. & 5 \\
3 mo prior to review & 32 \\
\hline 2 mo prior to review & $9(5)$ \\
\hline VA-supplied medications, mean (SD), No. & $6(9)$ \\
\hline Documentation of cognitive impairment, No. (\%) & $31(45)$ \\
\hline Mental health diagnosis, No. (\%) & \\
\hline
\end{tabular}

proportion of referrals that were completed at 3 months. ${ }^{4}$ As this quality improvement project was undertaken for programmatic evaluation, the University of WisconsinMadison Health Sciences Institutional Review Board determined that this project did not meet the federal definition of research and therefore review was not required. Data were analyzed using descriptive statistics.

\section{RESULTS}

A total of 78 veterans who had $\geq 1$ ACSCrelated hospitalization in the past year and who were categorized as low risk were identified, and 69 veterans were reviewed. Nine patients were not included based on hospice care and no longer receiving primary care through the facility. Eight patients were found to have optimized care with no further action warranted after review. Based on their assigned PACT, there was a range of 0 to 5 patients identified per team. Fifty-one patients were contacted, and 37 accepted $\geq 1$ referral. Most of the patients were white and male (Table). The most common ACSCs were hypertension (68\%), COPD (46\%), and T2DM (30\%); additional ACSCs included angina (18\%), pneumonia (15\%), UTIs (10\%), CHF (6\%), and asthma, dehydration, and perforated appendix 
FIGURE Accepted and Completed Referrals

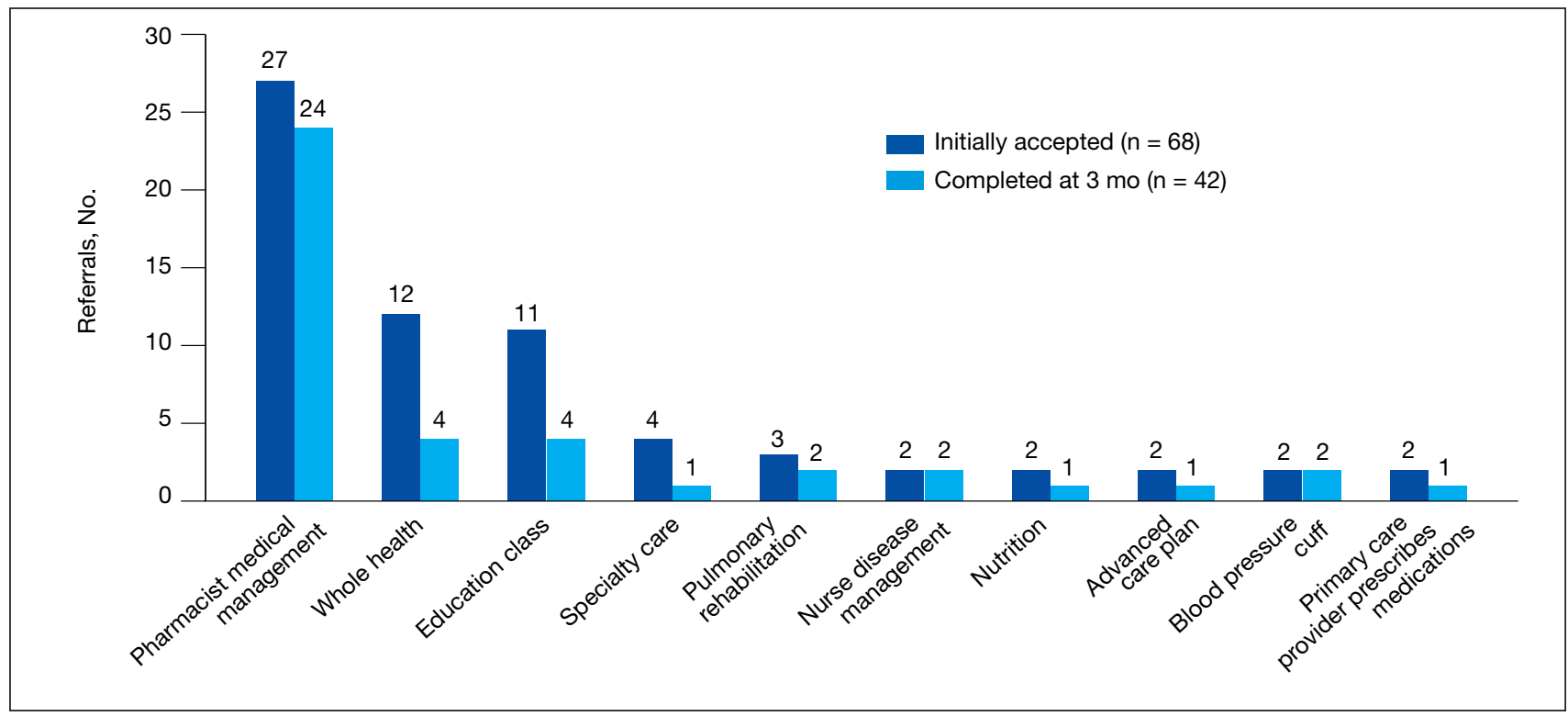

(1.5\% for each). Any ACSC listed as a diagnosis for a patient was included, regardless of whether it was related to a hospitalization. Most referrals were offered by pharmacists (pharmacy resident, 41\%; CPS, 29\%), followed by the nurse care manager $(18 \%)$ and the primary care provider (12\%). One patient passed away related to heart failure complications prior to being contacted to offer additional referrals. Of the 9 patients that were unable to be contacted, 4 did not respond to 3 phone call attempts and 5 had no documentation of referrals being offered after the initial chart review and recommendation was completed.

Most of the initially accepted referrals ( $\mathrm{n}=68$ ) were for CPS disease management, whole health/wellness, and educational classes (Figure). Of the 28 initially accepted referrals for CPS disease management, most were for COPD (10) and hypertension (8), followed by neuropathic pain (3), vitamin D deficiency (3), hyperlipidemia (2), and T2DM (2). At 3 months, all referrals were completed except for 1 hypertension, 1 vitamin $\mathrm{D}$ deficiency, and 2 hyperlipidemia referrals. There were 6 COPD, 4 T2DM selfmanagement, and 1 chronic pain class referrals made with 3 COPD and 1 T2DM referrals completed at 3 months. Two tobacco treatment and 2 palliative care referrals were specialty referrals accepted by patients with 1 palliative care referral completed at 3 months.

In terms of feasibility, the chart review took an average (SD) of 13 (4) minutes, and contacting the patient to offer referrals took an average of 8 (5) minutes. Most of the accepted referrals were completed by 3 months (42/68, 62\%).

Comparing the 3 months prior to and the 3 months after offering referrals, there was a cumulative quantitative decrease in the number of ED visits ( 5 to 1 ) and hospitalizations (11 to 5). The 1 ED visit was for a patient who was unable to be contacted to offer additional referrals as were 4 of the hospitalizations. One of the hospitalizations was for a patient who was deemed to have optimized care with no additional referrals necessary.

\section{DISCUSSION}

Evaluation of the review and referral process for patients at low risk for hospitalization from an ACSC was a proactive approach toward optimizing primary care for veterans, and the process increased patient access to education and primary care. There was a high initial patient acceptance rate of referrals and a high completion rate when offered by PACT members. Based on the number of identified patients, the time spent completing chart reviews and contacting patients to 
offer referrals for each PACT CPS and team was feasible to conduct.

As there were 69 eligible patients identified over a 3-month period for a single VA facility, including all community-based outpatient clinics serving an estimated 130,000 veterans, the additional time and workload for an individual PACT to reach out to these patients is minimal. Completing the review and outreach process for an average of 21 minutes per patient for at most 5 patients per primary care provider team is feasible to complete during the recommended 4 hours of weekly CPS population health management responsibilities.

\section{Limitations}

Several limitations were identified with the implementation of the project. A variety of PACT members completed initial outreach to veterans regarding additional referrals, which may have resulted in a lack of consistency in the approach and discussion of offering referrals to patients. Although there may be a difference in how the team members made referral offers to patients and therefore varying acceptance rates by patients, the process was thought to be more generalizable to the PACT approach for providing care in the VA. In addition, the time to contact patients to offer referrals was not always documented in the electronic health record, making the documented time an estimate. Given that patients identified were managed by a variety of PACT members, there were differences noted among PACTs in terms of acceptability of offering referrals to patients.

While there was a decrease noted in ED visits and hospitalizations when comparing 3 months before and afterward, additional data are needed to provide further insight into this relationship. As the patients identified were at low risk for hospitalization from an ACSC and had 1 or 2 hospitalizations within the year prior, additional time is warranted to compare 12-month ED visits and hospitalization rates postintervention. Finally, these findings may be limited in generalizability to other health care systems as the project was conducted among a specific, veteran patient population with PACT CPSs practicing independently within an established broad scope of practice.

\section{Future Directions}

Future directions include incorporating the review and referral process into the PACT CPS population health management responsibilities as a way to use all PACT members to enhance primary care delivered to veterans. To further elucidate the relationship between the referral process and hospitalization rates, a longer data collection period is needed.

\section{CONCLUSIONS}

Identifying patients at risk for hospitalization from an ACSC via a review and referral process by using the VA PACT structure and team members was feasible and led to increased patient access to primary care and additional services. The PACT CPS would benefit from using a similar approach for population health management for low risk for hospitalization patients or other identified chronic conditions.

\section{Acknowledgments}

Presented at the Wisconsin Pharmacy Residency Conference at the Pharmacy Society of Wisconsin Educational Conference April 10, 2019, in Madison, Wisconsin.

\section{Author disclosures}

The authors report no actual or potential conflicts of interest with regard to this article.

\section{Disclaimer}

The opinions expressed herein are those of the authors and do not necessarily reflect those of Federal Practitioner, Frontline Medical Communications Inc., the US Government, or any of its agencies.

\section{References}

1. US Department of Veterans Affairs, Veterans Health Administration, Pharmacy Benefits Management Service, Clinical Pharmacy Practice Office. Clinical pharmacy specialist (CPS) role in management of ambulatory care sensitive conditions (ACSC). [Nonpublic source.]

2. US Department of Health and Human Services, Agency for Healthcare Research and Quality. Guide to prevention quality indicators: hospital admission for ambulatory care sensitive conditions. https://www.ahrq.gov/downloads /pub/ahrqqi/pqiguide.pdf. Revised April 17, 2002. Accessed July 16, 2020.

3. Yoon J, Chang E, Rubenstein L, et al. Impact of primary care intensive management on high-risk veterans' costs and utilization. Ann Intern Med. 2018;168(12):846-854. doi:10.7326/M17-3039

4. Proctor E, Silmere H, Raghavan R, et al. Outcomes for implementation research: conceptual distinctions, measurement challenges, and research agenda. Adm Policy Ment Health. 2011;38:65-76. doi:10.1007/s10488-010-0319-7 